\title{
Control de potencia y velocidad de rotación de un aerogenerador usando controladores predictivos
}

\section{Power and rotational speed control of a wind turbine using predictive controllers}

\author{
Semaria Ruiz-Alvarez a \& Jairo Espinosa ${ }^{b}$ \\ a Facultad de Minas, Universidad Nacional de Colombia, Medellín, Colombia. seruizal@unal.edu.co \\ ${ }^{b}$ Facultad de Minas, Universidad Nacional de Colombia, Medellín, Colombia. jespinov@unal.edu.co
}

Recibido: junio 30, 2018. Aceptado: julio 30, 2018.

\begin{abstract}
Resumen
La estabilidad de los sistemas de potencia, en los cuales se implementan fuentes renovables como la eólica, es un área de gran atención en la actualidad ya que se requiere cumplir con una calidad en el servicio energético suministrado, y las fuentes renovables, ante su variabilidad en la potencia generada por la dependencia de las condiciones climáticas, pueden afectar dicha calidad. Por esta razón, es necesario aplicar estrategias de control que permitan realizar una integración de las fuentes renovables para tratar de minimizar los efectos adversos de estas sobre la red y que además admitan incluir los límites operativos de estas fuentes para evitar daños en ellas. En este trabajo se plantean dos estrategias de control, LQPC y GPC, para controlar la velocidad angular y el torque de un aerogenerador con el objetivo de garantizar un determinado valor para la potencia de salida de este y evitar variaciones bruscas en la velocidad angular que puedan generar desgaste o estrés mecánico.
\end{abstract}

Palabras Clave: Aerogenerador, Controlador LQPC, Controlador GPC, Regulación de frecuencia, Sistemas de potencia.

\begin{abstract}
The stability of the power systems is an area of great attention at present since it is required to comply with a determined quality standard in the supplied energy service. Such quality can be affected by the variability in power generated from renewable sources, due to the dependence on climatic and environmental conditions. For this reason, it is necessary to apply control strategies that allow integration of renewable sources while minimizing their adverse effects in the power grid. For the particular case of wind turbines, it is desirable to include their operational limits in control schemes to avoid damages for improper use. In this work, LQPC and GPC control strategies are proposed to control the angular speed and torque of a wind turbine. These schemes must guarantee a given reference value in output power, and to avoid sudden variations in the angular velocity that can generate wear or mechanical stress in wind turbines.
\end{abstract}

Keywords: Wind turbines, LQPC control, GPC control, Frequency regulation, Power systems.

\section{INTRODUCCIÓN}

El uso de la energía eléctrica juega un papel muy importante en el desarrollo socio-económico de la población [1]; por ello, existe una creciente demanda energética en zonas urbanas y rurales en el mundo que debe satisfacerse a través del incremento de la capacidad de generación [2]. Para el año 2012, aproximadamente el $68 \%$ de la energía generada en el mundo provenía de la quema de combustibles fósiles (gas natural y petróleo) [3]; sin embargo, estos combustibles son la principal causa de contaminación ambiental, uno de los fenómenos que genera cambio climático en el contexto mundial, además de que los recursos naturales de los cuales provienen los combustibles fósiles son limitados y en algún momento serán insuficientes para cubrir la demanda energética [4]. Por lo tanto, se ha convertido en una prioridad utilizar energías renovables [5]-[7].

Un factor clave que ha favorecido la inclusión de energías renovables en la red de potencia ha sido la tendencia a implementar generadores de energía eléctrica a pequeña escala que comúnmente están ubicados cerca de los usuarios finales, a lo cual se le ha denominado generación distribuida [8], [9]. Esta tendencia surge de que se puede lograr mayor eficiencia con la ubicación de pequeñas unidades de generación cerca a los usuarios finales, ya que no se tendría que transportar la energía desde las estaciones de generación centralizadas hasta los lugares de consumo remotos, lo que mejora la calidad de la potencia en el suministro y brinda mayor confiabilidad al reducir las emisiones de carbono si se emplean fuentes renovables [10]. Algunas de las fuentes de generación

Citar como:

S. Ruiz-Alvarez, J. Espinosa. “Control de potencia y velocidad de rotación de un aerogenerador usando controladores predictivos" Revista CINTEX, Vol. 23(1), pp. 60-76. 2018. 
distribuida más utilizadas son: las microturbinas, las celdas de combustible, los paneles fotovoltaicos, las turbinas eólicas y los bio-digestores, entre otros [11], [12].

Cuando estas fuentes son integradas en la red convencional existe un requerimiento para criterios de calidad de la potencia; por lo tanto, se debe regular la potencia generada de tal forma que estas no ocasionen problemas de estabilidad en el sistema [13]. Particularmente, en los generadores eólicos se plantea el control de la potencia generada con el objetivo de que estos contribuyan con la regulación de frecuencia en la red, como lo muestran varios trabajos reportados en la literatura [14], [15]. En [16], se propone una estructura óptima cuadrática de múltiples modelos para turbinas eólicas de velocidad variable, con el fin de proveer una reserva de potencia activa que favorezca la regulación primaria de frecuencia. Técnicas de control predictivo han sido aplicadas bajo un modelo reducido de turbinas de viento que incluye las contribuciones al control de frecuencia por parte de las unidades eólicas [17]. Los estudios reportados en [18] y [19] se han enfocado en el análisis de sensibilidad de las turbinas de viento de velocidad variable y sus impactos en la estructura tradicional de regulación de la frecuencia en sistemas de potencia. Las técnicas de respuesta inercial de las unidades eólicas, a través de la modificación del algoritmo de extracción de máxima potencia del viento también han sido exploradas en [20], operando en modo "descargado" (deloaded) o por debajo del punto óptimo de potencia activa extraída [21], [22]. También se debe mencionar que los autores han abordado esta problemática previamente a través de la implementación de estructuras de reguladores cuadráticos lineales para mantener las contribuciones de las unidades eólicas a la regulación primaria, partiendo de modelos de emulación inercial [23] y, adicionalmente, con la minimización de los cambios en la velocidad de los aerogeneradores para evitar daños mecánicos [24].

En este trabajo se realizará un análisis de la implementación de controladores LQPC (Linear Quadratic Predictive Control) [25] y GPC (Generalized Predictive Control) [26] de horizonte de predicción finito e infinito para controlar la potencia y velocidad de rotación de un aerogenerador. En ambos controladores, además del control de potencia y velocidad, se mantendrá el punto de operación dentro de los límites permitidos para las variables del aerogenerador.

\section{MODELO DE AEROGENERADOR}

\subsection{Descripción del modelo}

En esta sección se presenta el modelo en espacio de estados de una turbina eólica de velocidad variable, como se describió en [27]:

$$
\begin{gathered}
\dot{w}_{r}=\frac{P_{r}\left(w_{r}, \theta, v\right)}{w_{r} J_{r}}-\frac{w_{r} D_{s}}{J_{r}}+\frac{w_{g} D_{s}}{J_{r} N_{g}}-\frac{\delta K_{s}}{J_{r}} \\
\dot{w}_{g}=\frac{w_{r} D_{s}}{N_{g} J_{g}}-\frac{w_{g} D_{s}}{N_{g}^{2} J_{g}}+\frac{\delta K_{s}}{N_{g} J_{g}}-\frac{T_{g}}{J_{g}} \\
\dot{\delta}=w_{r}-\frac{w_{g}}{N_{g}} \\
\dot{\theta}=-\frac{1}{\tau_{\theta}} \theta+\frac{1}{\tau_{\theta}} \theta_{r} \\
\dot{T}_{g}=-\frac{1}{\tau_{t}} T_{g}+\frac{1}{\tau_{t}} T_{g, r}
\end{gathered}
$$

Se definen los siguientes estados, entradas y salidas:

$$
x=\left[\begin{array}{lllll}
w_{r} & w_{g} & \delta & \theta & T_{g}
\end{array}\right]^{T} ; u=\left[\begin{array}{ll}
\theta_{r} & T_{g, r}
\end{array}\right]^{T} ; y=\left[\begin{array}{ll}
w_{r} & P_{g}
\end{array}\right]^{T}
$$

Se toma la velocidad del viento $v$ como una perturbación en el sistema ya que esta varía cambiando la potencia generada por este. También se define como salida la potencia eléctrica generada, la cual está dada por la siguiente expresión:

$$
P_{e}=T_{g} w_{g}
$$

Los valores de los parámetros del sistema son los siguientes: Densidad del aire $\rho=1.2\left[\mathrm{~kg} / \mathrm{m}^{3}\right]$; Inercia del rotor $J_{r}=90000\left[\mathrm{~kg} \cdot \mathrm{m}^{2}\right]$; Inercia del generador $J_{g}=10\left[\mathrm{~kg} \cdot \mathrm{m}^{2}\right]$; Constante de resorte del sistema de transmisión $K_{s}=8 e 6[\mathrm{~N} / \mathrm{m}]$; Coeficiente de amortiguamiento del sistema de transmisión $D_{s}=8 e 4\left[\mathrm{~s}^{-1}\right]$; 
Relación del engranaje $N_{g}=24.6$; Longitud del aspa $R=14.5[\mathrm{~m}]$; Constante de tiempo del actuador para el cambio de ángulo de las aspa $\tau_{\theta}=0.15[\mathrm{~s}] \mathrm{s} ; \tau_{T g}=0.1$ [s] es la constante de tiempo del torque generador [28].

\subsection{Linealización}

Como el objetivo del controlador es mantener la velocidad de rotación constante y al mismo tiempo proveer una potencia de salida constante, hay tres parámetros que se conocen: $w_{r, n o m}=4.29 \frac{\mathrm{rad}}{\mathrm{s}} ; w_{\mathrm{g}, \mathrm{nom}}=$ $103.9 \frac{\mathrm{rad}}{\mathrm{s}} ; P_{\text {,nom }}=225000 \mathrm{~W}$. Adicionalmente, para una velocidad de viento dada $\mathrm{v}$, pueden calcularse los valores de estado estacionario de las demás variables haciendo las dinámicas cero, $\dot{x}=0$. Para ello, se debe despejar la variable $\theta$ de la ecuación no lineal:

$$
\frac{P_{r}\left(w_{r}, \theta, v\right)}{w_{r} J_{r}}-\frac{w_{r} D_{s}}{J_{r}}+\frac{w_{g} D_{s}}{J_{r} N_{g}}-\frac{\delta K_{s}}{J_{r}}=w_{r, \text { nom }}
$$

Teniendo en cuenta que $T_{g}=\frac{P_{g}}{w_{g}}$, si se asume una velocidad promedio del viento de $18 \mathrm{~m} / \mathrm{s}$ y se resuelve la anterior ecuación no lineal se obtienen los valores para las variables de estado descritos en la tabla I.

TABLA I. PUNTO DE EQUILIBRIO DEL SISTEMA

\begin{tabular}{|c|c|c|c|c|c|}
\hline$v[\mathrm{~m} / \mathrm{s}]$ & $w_{r, \text { nom }}[\mathrm{rad} / \mathrm{s}]$ & $w_{g, \text { nom }}[\mathrm{rad} / \mathrm{s}]$ & $\delta[\mathrm{rad}]$ & $\theta[\mathrm{deg}]$ & $T_{g}[\mathrm{Nm}]$ \\
\hline 18 & 4.20 & 103.9 & 0.00655 & 19.1 & 2130 \\
\hline
\end{tabular}

Después de obtener el punto de equilibrio del sistema se procede a realizar la linealización alrededor de este punto, de la forma descrita a continuación:

$$
\begin{aligned}
& \dot{\Delta x}=A \Delta x+B \Delta u \\
& \Delta y=C \Delta x+D \Delta u
\end{aligned}
$$

Donde $x^{*}$ es el punto de equilibrio del sistema; $u_{s s}$ es el valor en estado estacionario de las entradas del sistema; $d_{s s}$ es el valor en estado estacionario de las perturbaciones del sistema; $y^{*}$ es el valor de la salida al evaluar los estados en el punto de equilibrio y las entradas en el valor de estado estacionario. Además, $\Delta x=x-x^{*} ; \Delta u=$ $u-u_{s s} ; \Delta y=y-y^{*}$.

\subsection{Límites en las variables del aerogenerador}

TABLA II. VALORES LÍMITE PARA LOS ESTADOS DEL SISTEMA

\begin{tabular}{|c|c|c|}
\hline Variable & $\begin{array}{c}\text { Valor } \\
\text { máximo }\end{array}$ & Valor mínimo \\
\hline$w_{r}[\mathrm{rad} / \mathrm{s}]$ & 6.45 & 0 \\
\hline$w_{g}[\mathrm{rad} / \mathrm{s}]$ & 160.43 & 0 \\
\hline$\delta[\mathrm{rad}]$ & 0.17 & 0 \\
\hline$\theta[\mathrm{deg}]$ & 25 & -1 \\
\hline$T_{g}[\mathrm{~N} \mathrm{~m}]$ & 2556 & 0 \\
\hline$\theta_{r}[\mathrm{deg}]$ & 25 & -1 \\
\hline$T_{g, r}[\mathrm{~N} \mathrm{~m}]$ & 2556 & 0 \\
\hline$w_{r}[\mathrm{rad} / \mathrm{s}]$ & 6.45 & 0 \\
\hline$P_{g}[\mathrm{w}]$ & 332022.42 & 0 \\
\hline
\end{tabular}

La tabla II presenta los valores máximos y mínimos entre los cuales pueden operar los estados, las entradas y las salidas del aerogenerador. Los valores máximos para las salidas y los mínimos para los estados $w_{r}, w_{g}$ y $\theta$ se tomaron de [29]. Para la variable $\delta$ se asumió un valor mínimo de 0 [rad], dado que, al tratarse de la diferencia de ángulo entre el rotor y el generador, se restringió a que fuese positivo y que tuviese un valor máximo de 0.1745 ; 
esta última cifra se tomó con el criterio de que debería estar entre 0 y $2 \pi$, y ser lo suficientemente pequeño para evitar efectos de desenganche en el generador [29]. Además, se asumió que el torque máximo del generador es 1,2 veces su valor nominal. Los valores máximos y mínimos de las entradas $\theta_{r}, T_{g, r}$ se tomaron iguales a los valores límite de los estados $\theta$ y $T_{g}$. El valor máximo de la salida $P_{g}$ se tomó como 1.5 veces el valor de la potencia nominal, y el valor mínimo se estableció en 0 . Todos los controladores se trabajaron con un tiempo de discretización $d_{1}$ de 0.1 segundos, y un horizonte $\mathrm{N}=20$.

\section{CONTROLADOR LQPC (LINEAR QUADRATIC PREDICTIVE CONTROL)}

Sea el sistema lineal discreto de la forma:

$$
\begin{gathered}
x(k+1)=A x(k)+B u(k) \\
y(k)=C x(k)+D u(k)
\end{gathered}
$$

Si se elige un horizonte de predicción $N$, el sistema en la ecuación (6) puede expresarse de la forma:

$$
\begin{gathered}
x_{k}=\Psi x(k+j)+\bar{\gamma} u_{k}, \text { donde } \\
x_{k}=\left[\begin{array}{c}
y(k+j+1) \\
\vdots \\
y(k+j+N)
\end{array}\right], u_{k}=\left[\begin{array}{c}
u(k+j) \\
\vdots \\
u(k+j+N)
\end{array}\right] \\
\Psi=\left[\begin{array}{c}
A \\
\vdots \\
A^{N}
\end{array}\right], \gamma=\left[\begin{array}{ccc}
B & \cdots & 0 \\
\vdots & \ddots & \vdots \\
B A^{N-1} & \cdots & B
\end{array}\right], \bar{\gamma}=[\gamma \mid 0]
\end{gathered}
$$

Así, se calcula la acción de control que minimice la siguiente función de costo, teniendo en cuenta que los valores de los estados y las entradas se deben encontrar entre los valores máximos y mínimos permitidos.

Sujeto a

$$
J=\sum_{j=1}^{N} x(k+j)^{T} Q x(k+j)+\sum_{j=0}^{N} u(k+j)^{T} R u(k+j)
$$

$$
\begin{gathered}
x(k+j+1)=A x(k+j)+B u(k+j) \\
u_{\min } \leq u(k+j) \leq u_{\max } \\
x_{\min } \leq x(k+j) \leq x_{\max }
\end{gathered}
$$

Teniendo en cuenta el planteamiento anterior, se busca llevar el problema de optimización a un problema de programación cuadrática, para garantizar convexidad en la función de costo y así poder encontrar el mínimo global del problema, esto se hace reemplazando la expresión para $x_{k}$ en la función objetivo; por lo tanto, se obtiene:

Donde:

$$
J\left(u_{k}\right)=u_{k}^{T} H u_{k}+2 F u_{k}+r
$$

$$
\begin{gathered}
H=\bar{\gamma}^{T}\left[\begin{array}{ccc}
Q & \cdots & 0 \\
\vdots & \ddots & \vdots \\
0 & \cdots & Q
\end{array}\right] \bar{\gamma}+\left[\begin{array}{ccc}
R & \cdots & 0 \\
\vdots & \ddots & \vdots \\
0 & \cdots & R
\end{array}\right] \\
F=2(\Psi x(k+j))^{T}\left[\begin{array}{ccc}
Q & \cdots & 0 \\
\vdots & \ddots & \vdots \\
0 & \cdots & Q
\end{array}\right] \bar{\gamma} \\
r=(\Psi x(k+j))^{T}\left[\begin{array}{ccc}
Q & \cdots & 0 \\
\vdots & \ddots & \vdots \\
0 & \cdots & Q
\end{array}\right](\Psi x(k+j))
\end{gathered}
$$

Así, el problema de optimización puede plantearse nuevamente como:

$$
\min \left(u_{k}^{T} H u_{k}+2 F u_{k}+r\right)
$$

Sujeto a:

$$
\begin{aligned}
& \bar{\gamma} u_{k} \leq \overline{x_{\max }}-\Psi x(k+j) \\
& -\bar{\gamma} u_{k} \leq \Psi x(k+j)-\overline{x_{m i n}}
\end{aligned}
$$


Control de potencia y velocidad de rotación de un aerogenerador usando controladores predictivos Control of power and rotation speed of a wind turbine using predictive controllers

$$
\begin{aligned}
& I u_{k} \leq \overline{u_{\max }} \\
& -I u_{k} \leq \overline{u_{m i n}}
\end{aligned}
$$

Donde $\overline{x_{\max }}$ es un vector que contiene la extensión del valor máximo de los estados $x_{\max }, \mathrm{N}$ veces; $\overline{x_{m} ı n}$ es un vector que contiene la extensión del valor mínimo de los estados $x_{\min }, \mathrm{N}$ veces; $\overline{u_{\max }}$ es un vector que contiene la extensión del valor máximo de las entradas $u_{\text {max }}, \mathrm{N}+1$ veces y $\overline{u_{\min }}$ es un vector que contiene la extensión del valor mínimo de las entradas $u_{m i n}, \mathrm{~N}+1$ veces.

Para el presente trabajo, el problema en (10) se resuelve con la rutina quadprog en Matlab. A continuación, se presentan los resultados obtenidos mediante simulación en Matlab, aplicando la acción de control $u_{k}$, obtenida con la optimización anteriormente planteada, a la planta no lineal, para la cual se asume que los estados no son medibles y se emplea un filtro de Kalman para realizar su estimación.

\subsection{Respuesta del control LQPC}

En la Figura 2 se observa cómo, a partir de aproximadamente 10 segundos, ambas salidas $w_{r}$ y $P_{g}$ tienden a su valor de estado estacionario $(4.224 \mathrm{rad} / \mathrm{s}, 221348.28 \mathrm{w})$, teniendo en cuenta que iniciaron en un punto ligeramente diferente del valor de estado estacionario $\left(w_{r}=4.254 \mathrm{rad} / \mathrm{s}, T_{g}=2130.8 \mathrm{~N} \mathrm{~m}\right)$.
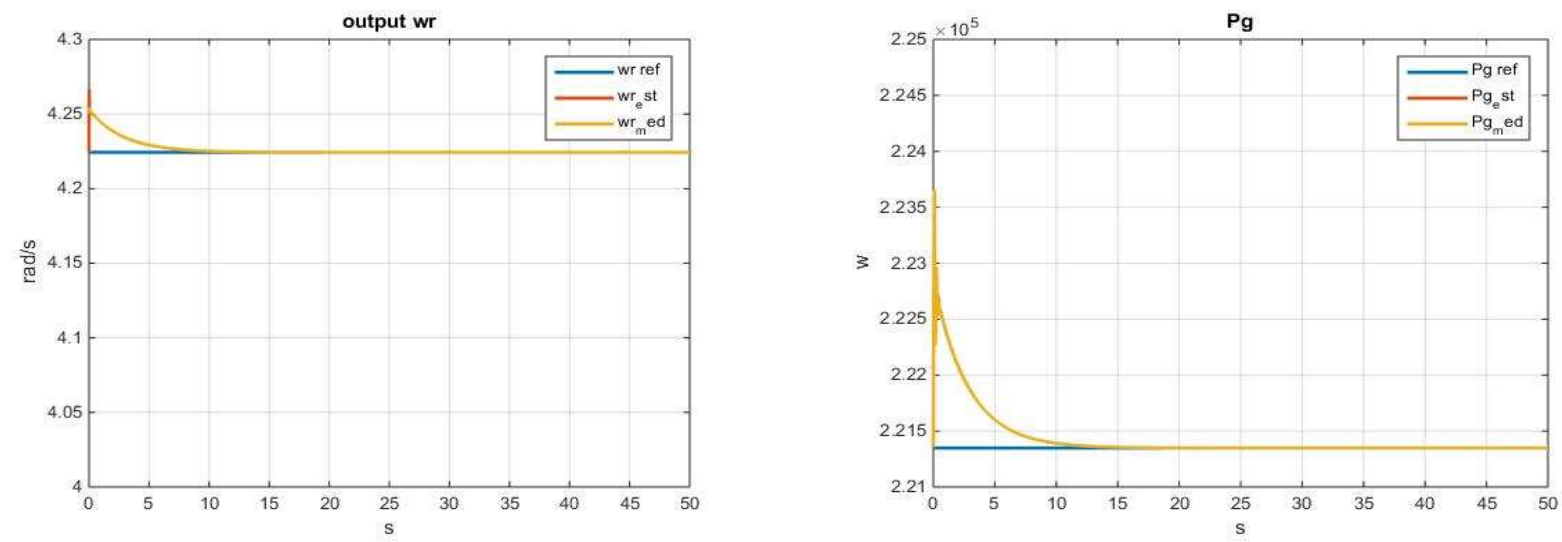

Figura 2. Respuestas de las salidas del sistema $w_{r}$ (izquierda) y $P_{g}$ (derecha) con control LQPC y valor inicial cercano al valor de estado estacionario
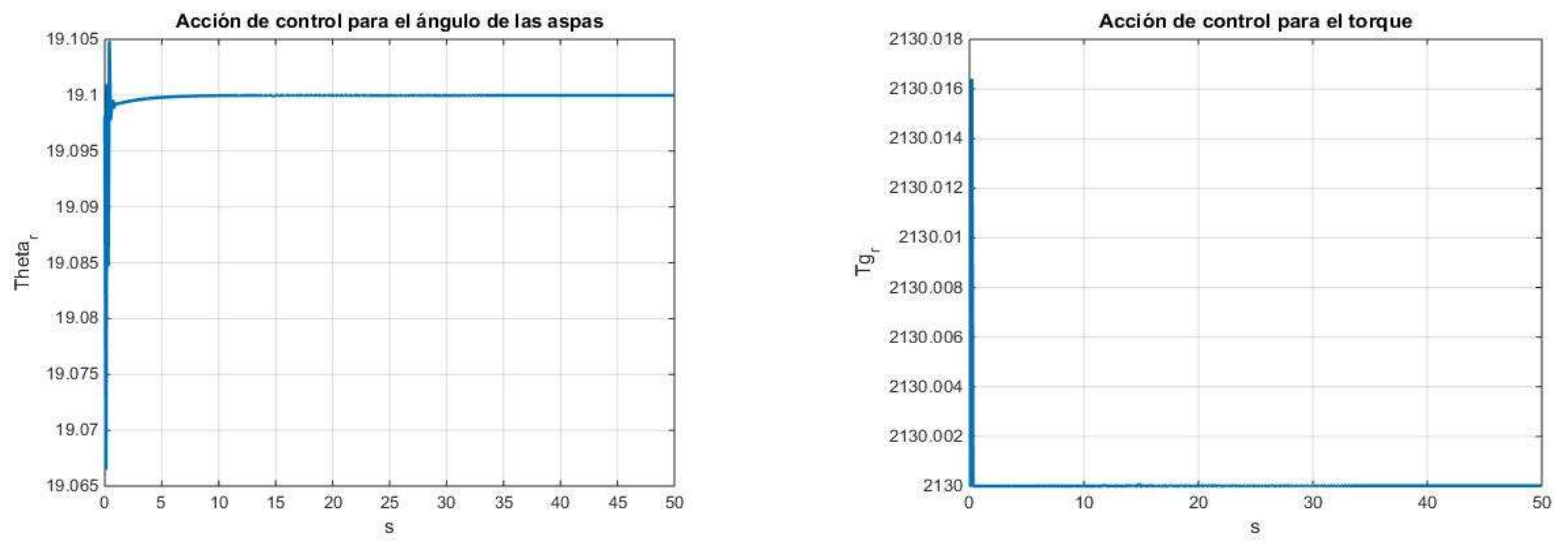

Figura 3. Respuestas de las acciones de control $\theta$ (izquierda) y $T_{g, r}$ (derecha) con control LQPC y valor inicial cercano al valor de estado estacionario 


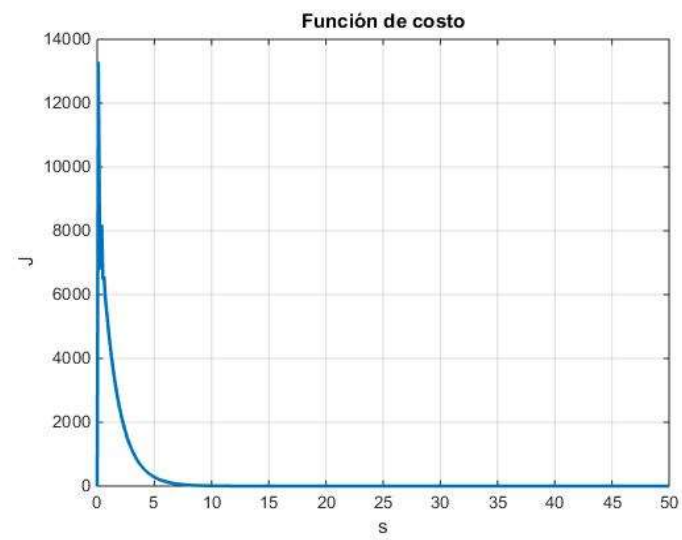

Figura 4. Funcional de costo para el control LQPC con valor inicial cercano al valor de estado estacionario

La Figura 3 muestra las respuestas de las acciones de control. Puede decirse que los cambios en las acciones de control afectan la respuesta del funcional de costo, tal como se observa en la Figura 4: inicialmente, hay un alto valor en la respuesta del funcional; ; sin embargo, a medida que las acciones de control tienden a cero, la función de costo también se va a cero, o a un valor mínimo (valor del residuo $r$ ). Este comportamiento era de esperarse, teniendo en cuenta que la ecuación para la función de costo dada por la ecuación (11), donde el término $r$ tiende a cero para este caso:

$$
J\left(u_{k}\right)=u_{k}^{T} H u_{k}+2 F u_{k}+r .
$$

Los cambios del punto inicial anteriores corresponden a un $8 \%$ y $5 \%$ de los valores de estado estacionario para $w_{r}$ y $T_{g}$ respectivamente, por lo cual se observa que, para cambios relativamente pequeños respecto al punto de inicial, el controlador LQPC logra estabilizar el sistema en su punto de equilibrio; sin embargo, esto tiene un impacto en la función de costo puesto que mientras más diferente sea este punto inicial del punto de equilibrio, las acciones de control necesarias para estabilizar el sistema deberán ser más bruscas o de mayor magnitud, lo cual ocasionará un alza en la función de costo.
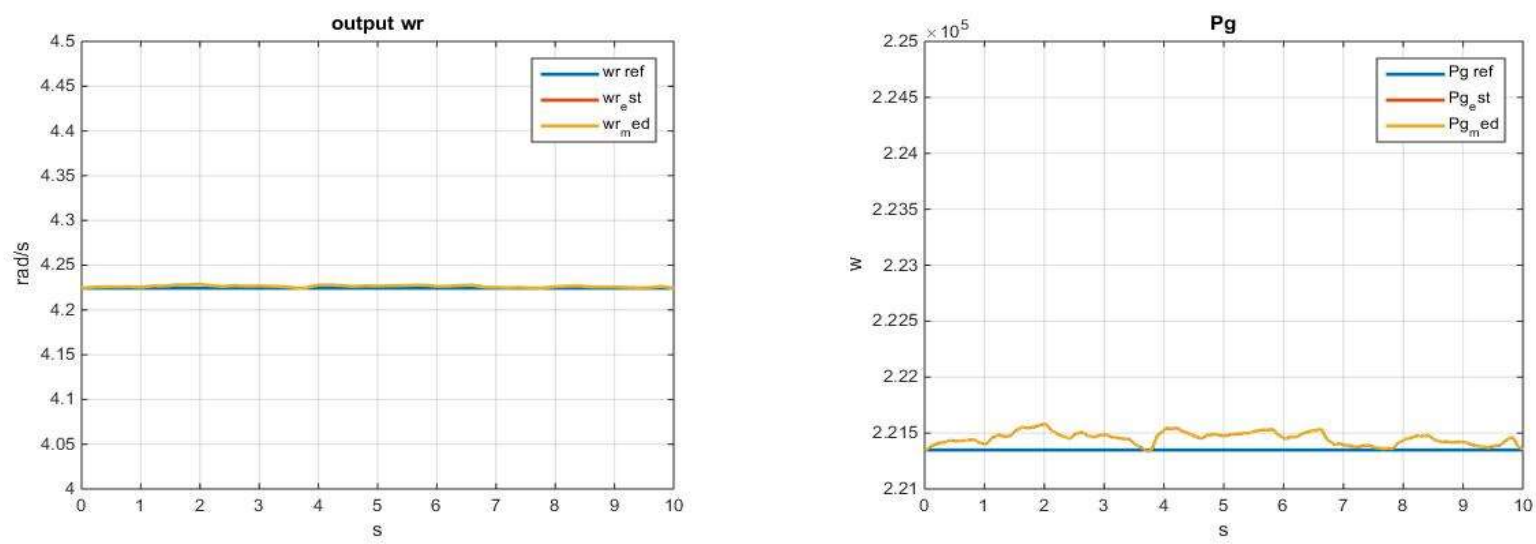

Figura 5. Respuestas de las salidas del sistema $w_{r}$ (izquierda) y $P_{g}$ (derecha) con control LQPC con perturbaciones 
Control de potencia y velocidad de rotación de un aerogenerador usando controladores predictivos Control of power and rotation speed of a wind turbine using predictive controllers
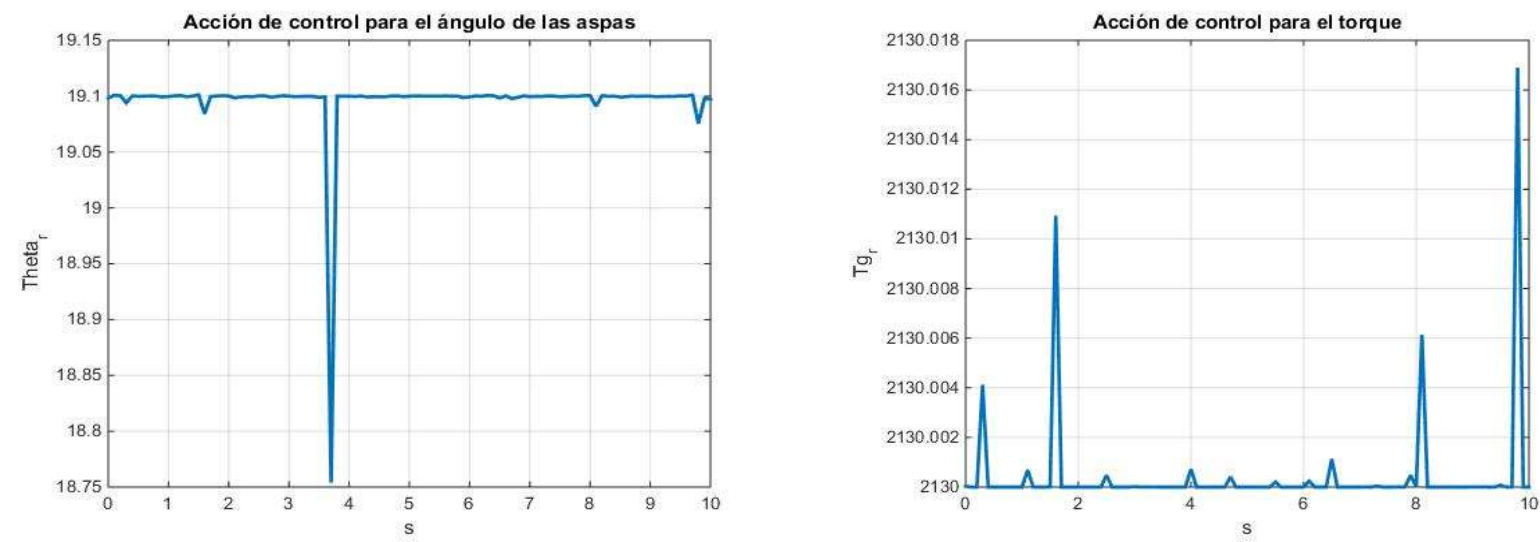

Figura 6. Respuestas de las acciones de control $\theta$ (izquierda) y $T_{g, r}$ (derecha) con control LQPC con perturbaciones

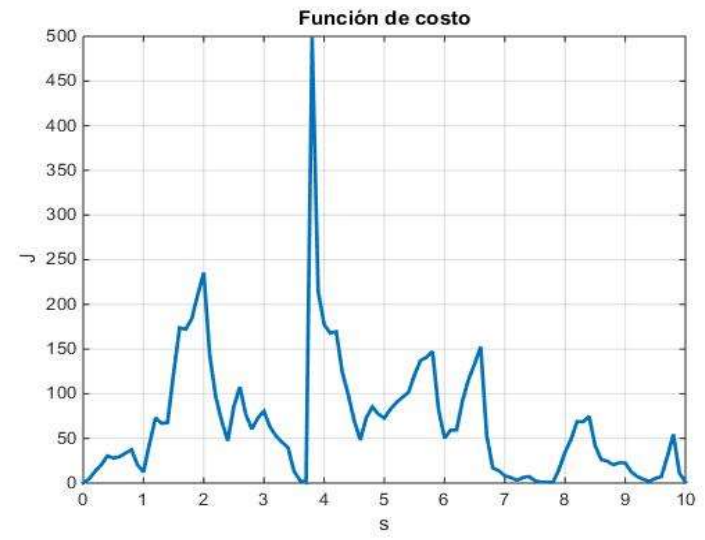

Figura 7. Funcional de costo para el control LQPC con perturbaciones

En la Figura 5 se puede observar cómo el controlador LQPC puede regular la potencia de salida y la velocidad del rotor ante cambios en la velocidad del viento con un valor medio de $18 \mathrm{~m} / \mathrm{s}$ y una desviación estándar de 0.001. Las acciones de control vistas en la Figura 6 deben compensar las perturbaciones, las cuales pueden aparecer en cualquier instante de tiempo. Nótese como la función de costo de la Figura 7 no tiende a cero en este caso con el paso del tiempo, a diferencia del caso anterior.

\subsection{Respuesta del control LQPC con restricciones adicionales}

Si se incluye una nueva restricción para la entrada del LQPC que limite los cambios bruscos en ella, se tiene:

$$
\begin{gathered}
|\Delta u(k+j)| \leq \Delta u_{\max } \\
-\Delta u_{\max } \leq \Delta u(k+j) \leq \Delta u_{\max }
\end{gathered}
$$

Expresando $\Delta u(k+j)$ en términos de $u_{k}$, se tiene:

Entonces:

$$
\left[\begin{array}{c}
u(k+j) \\
\vdots \\
u(k+j+N)
\end{array}\right]=\left[\begin{array}{c}
I \\
\vdots \\
I
\end{array}\right] u(k+j-1)+\left[\begin{array}{ccc}
I & \cdots & 0 \\
\vdots & \ddots & \vdots \\
I & \cdots & I
\end{array}\right]\left[\begin{array}{c}
\Delta u(k+j) \\
\vdots \\
\Delta u(k+j+N)
\end{array}\right]
$$

$$
\left[\begin{array}{c}
\Delta u(k+j) \\
\vdots \\
\Delta u(k+j+N)
\end{array}\right]=\left[\begin{array}{ccc}
I & \cdots & 0 \\
\vdots & \ddots & \vdots \\
I & \cdots & I
\end{array}\right]^{-1}\left[\begin{array}{c}
u(k+j) \\
\vdots \\
u(k+j+N)
\end{array}\right]-\left[\begin{array}{ccc}
I & \cdots & 0 \\
\vdots & \ddots & \vdots \\
I & \cdots & I
\end{array}\right]^{-1}\left[\begin{array}{c}
I \\
\vdots \\
I
\end{array}\right] u(k+j-1)
$$

Así, se tiene la siguiente restricción:

$$
\left[\begin{array}{ccc}
I & \cdots & 0 \\
\vdots & \ddots & \vdots \\
I & \cdots & I
\end{array}\right]^{-1} u_{k}-\left[\begin{array}{ccc}
I & \cdots & 0 \\
\vdots & \ddots & \vdots \\
I & \cdots & I
\end{array}\right]^{-1}\left[\begin{array}{c}
I \\
\vdots \\
I
\end{array}\right] u(k+j-1) \leq \Delta u_{\max }
$$




$$
-\left[\begin{array}{ccc}
I & \cdots & 0 \\
\vdots & \ddots & \vdots \\
I & \cdots & I
\end{array}\right]^{-1} u_{k}+\left[\begin{array}{ccc}
I & \cdots & 0 \\
\vdots & \ddots & \vdots \\
I & \cdots & I
\end{array}\right]^{-1}\left[\begin{array}{c}
I \\
\vdots \\
I
\end{array}\right] u(k+j-1) \leq-\Delta u_{\max }
$$

Implementando esta nueva restricción en el sistema con $\Delta u_{\max }=\left[\begin{array}{lll}0.001 & 0.2\end{array}\right]$, se obtienen las respuestas presentadas en la Figura, con las acciones de control para el ángulo de las aspas $\theta$ y el torque $T_{g, r}$ en la Figura y la Figura 10, respectivamente. En tales gráficas, puede observarse cómo la adición de la restricción para el cambio en la entrada causa que las acciones de control se hagan más suaves; por ejemplo, en el caso de la acción de control para el ángulo de las aspas $\theta$, al imponer la restricción sobre el $\Delta u$, el cambio más brusco en la acción de control, pasó de ser 0.003 a ser de 0.0007 , lo cual puede ser beneficioso para un sistema real, ya que generalmente los cambios abruptos en las entradas son costosos o difíciles de lograr y pueden ocasionar desgaste en el sistema.
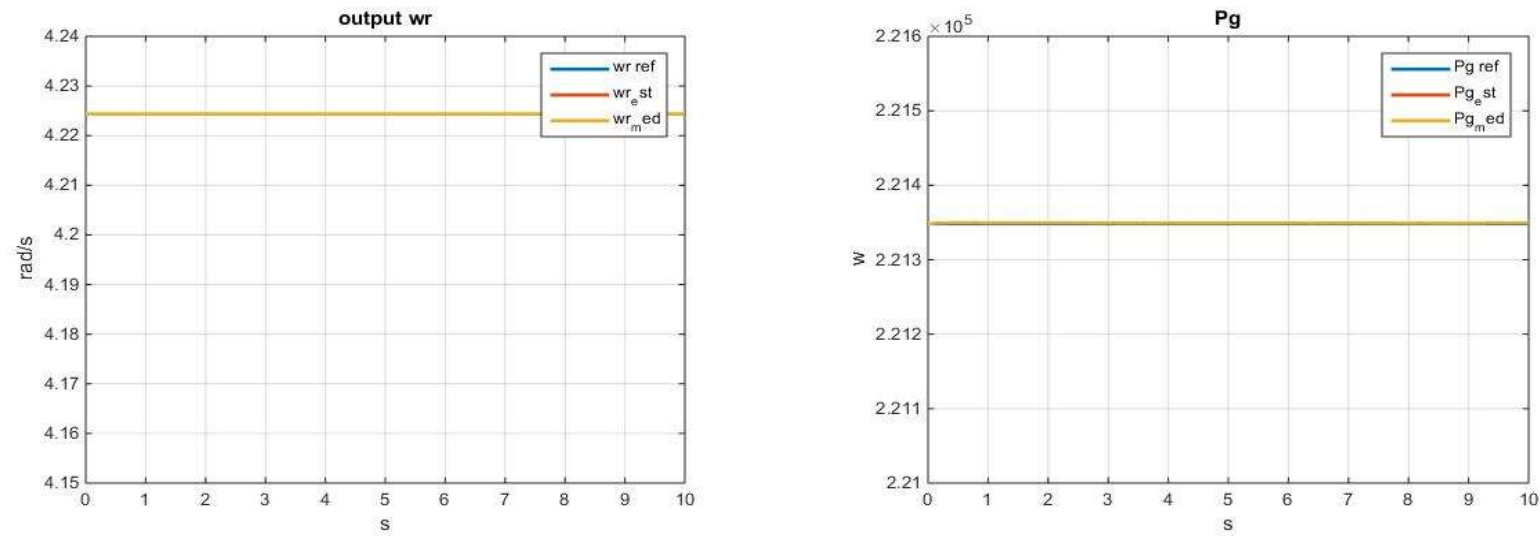

Figura 8. Respuestas de las salidas del sistema $w_{r}$ (izquierda) y $P_{g}$ (derecha) con control LQPC con restricciones adicionales
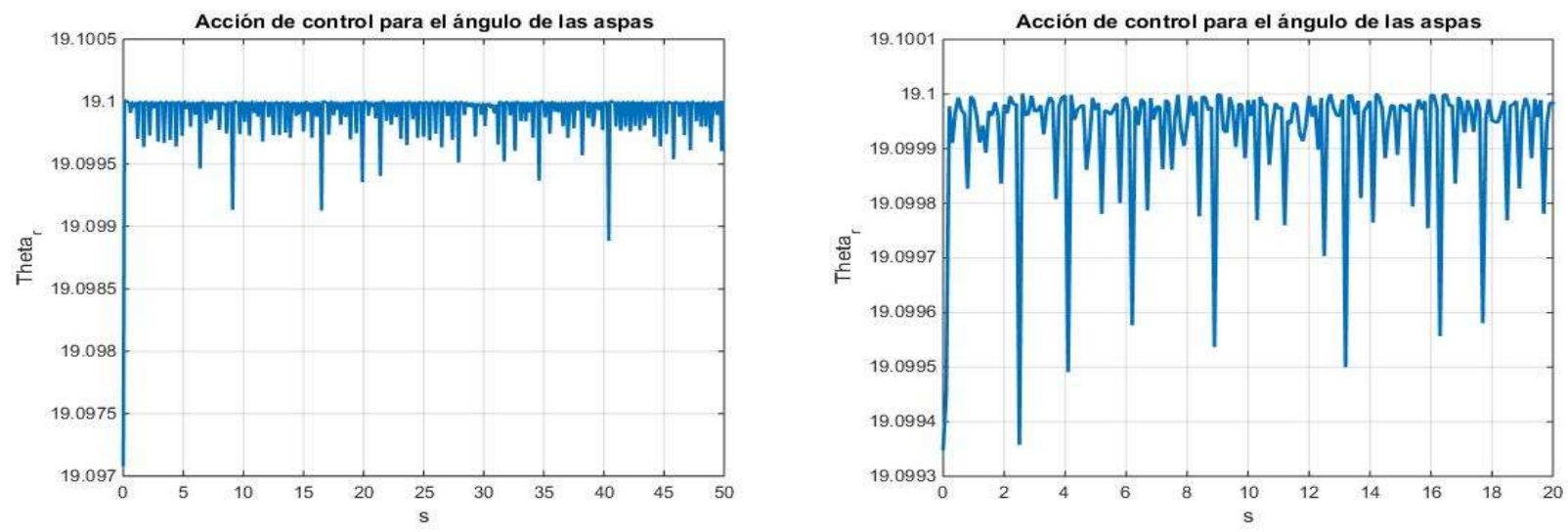

Figura 9. Respuestas de la acción de control $\theta$ para el LQPC: Sin restricción en $\Delta u$ (izq.) y Con restricción en $\Delta u$. (der.) 

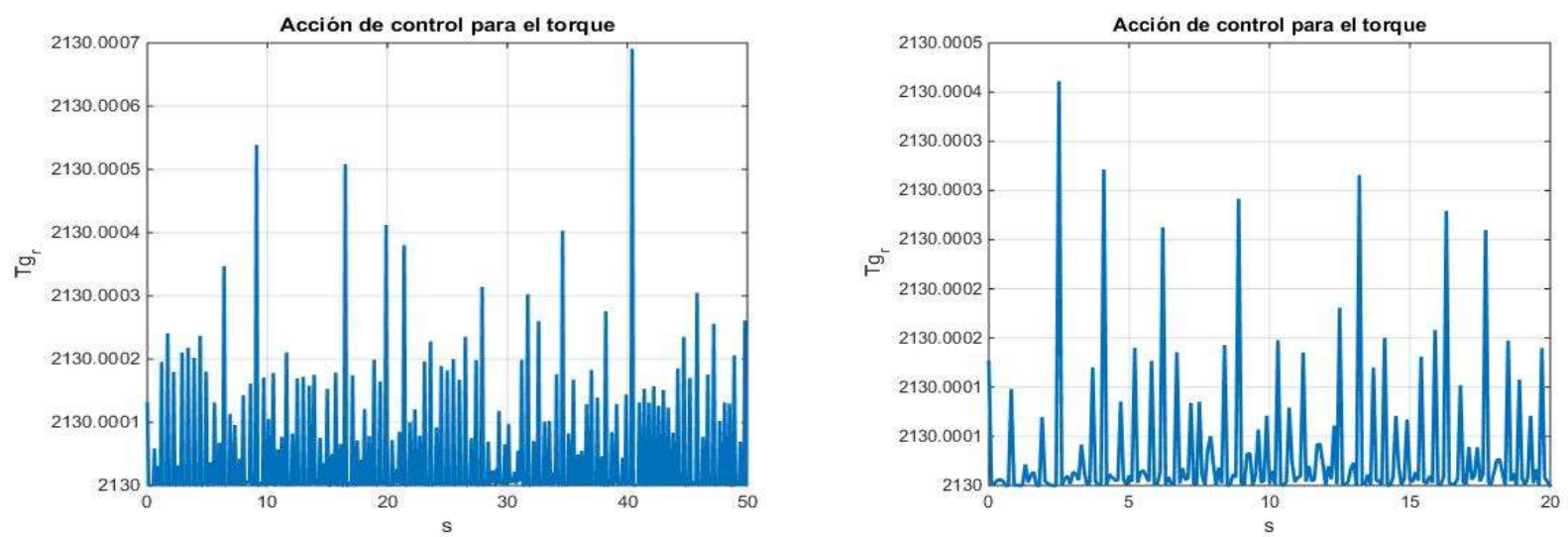

Figura 10. Respuestas de la acción de control $T_{g, r}$ para el LQPC: Sin restricción en $\Delta u$ (izq.) y Con restricción en $\Delta u$. (der.)

\section{CONTROLADOR GPC (GENERALIZED PREDICTIVE CONTROL)}

Consideremos nuevamente el sistema lineal discreto de la ecuación (6). Utilizando un horizonte de predicción, $\mathrm{N}$, dicho sistema se puede expresar de la forma:

Donde:

$$
y=\Gamma x(k+j)+\Delta u_{k}
$$

$$
\begin{gathered}
\Gamma=\left[\begin{array}{ccc}
C & \cdots & 0 \\
\vdots & \ddots & \vdots \\
0 & \cdots & C
\end{array}\right]\left[\begin{array}{c}
A \\
\vdots \\
A^{N}
\end{array}\right] \\
\Delta=\left[\begin{array}{ccc}
C & \cdots & 0 \\
\vdots & \ddots & \vdots \\
0 & \cdots & C
\end{array}\right]\left[\begin{array}{ccc}
B & \cdots & 0 \\
\vdots & \ddots & \vdots \\
B A^{N-1} & \cdots & B
\end{array}\right]\left[\begin{array}{ll}
I & 0
\end{array}\right]+\left[\begin{array}{ccc}
C & \cdots & 0 \\
\vdots & \ddots & \vdots \\
0 & \cdots & C
\end{array}\right]\left[\begin{array}{ll}
0 & I
\end{array}\right]
\end{gathered}
$$

Así, se calcula la acción de control que minimice la siguiente función de costo, teniendo en cuenta que los valores de los estados y las entradas se deben encontrar entre los valores máximos y mínimos permitidos:

Sujeto a

$$
J=\sum_{j=1}^{N}(y(k)-y r e f)^{T} Q(y(k)-y r e f)+\sum_{j=0}^{N} u(k+j)^{T} R u(k+j)
$$

$$
\begin{gathered}
x(k+j+1)=A x(k+j)+B u(k+j) \\
y(k+j)=C x(k+j)+D u(k+j) \\
u_{\min } \leq u(k+j) \leq u_{\max } \\
x_{\min } \leq x(k+j) \leq x_{\max }
\end{gathered}
$$

Llevando el problema anterior a una programación cuadrática, se tiene que:

Donde:

$$
y=\Gamma x+\Delta U
$$

$$
\begin{gathered}
\Gamma=\left[\begin{array}{ccc}
C & \cdots & 0 \\
\vdots & \ddots & \vdots \\
0 & \cdots & C
\end{array}\right]\left[\begin{array}{c}
A \\
\vdots \\
A^{N}
\end{array}\right] \\
\Delta=\left[\begin{array}{ccc}
C & \cdots & 0 \\
\vdots & \ddots & \vdots \\
0 & \cdots & C
\end{array}\right]\left[\begin{array}{ccc}
B & \cdots & 0 \\
\vdots & \ddots & \vdots \\
B A^{N-1} & \cdots & B
\end{array}\right]\left[\begin{array}{ll}
I & 0
\end{array}\right]+\left[\begin{array}{ccc}
C & \cdots & 0 \\
\vdots & \ddots & \vdots \\
0 & \cdots & C
\end{array}\right]\left[\begin{array}{ll}
0 & I
\end{array}\right]
\end{gathered}
$$

Así,

Donde:

$$
J\left(u_{k}\right)=u_{k}^{T} H u_{k}+2 F u_{k}+r
$$




$$
\begin{gathered}
H=\Delta^{T}\left[\begin{array}{ccc}
Q & \cdots & 0 \\
\vdots & \ddots & \vdots \\
0 & \cdots & Q
\end{array}\right] \Delta+\left[\begin{array}{ccc}
R & \cdots & 0 \\
\vdots & \ddots & \vdots \\
0 & \cdots & R
\end{array}\right] \\
F=2\left(\Gamma x(k+j)-\overline{y_{\text {ref }}}\right)^{T}\left[\begin{array}{ccc}
Q & \cdots & 0 \\
\vdots & \ddots & \vdots \\
0 & \cdots & Q
\end{array}\right] \Delta \\
r=\left(\Gamma x(k+j)-\overline{y_{r e f}}\right)^{T}\left[\begin{array}{ccc}
Q & \cdots & 0 \\
\vdots & \ddots & \vdots \\
0 & \cdots & Q
\end{array}\right]\left(\Gamma x(k+j)-\overline{y_{r e f}}\right)
\end{gathered}
$$

De esta manera, el problema de optimización se puede plantear nuevamente como:

$$
\min \left(u_{k}^{T} H u_{k}+2 F u_{k}+r\right)
$$

Sujeto a:

$$
\begin{gathered}
\Delta u_{k} \leq \overline{y_{\max }}-\Gamma x(k+j) \\
-\Delta u_{k} \leq \Gamma x(k+j)-\overline{y_{m i n}} \\
I u_{k} \leq \overline{u_{\max }} \\
-I u_{k} \leq \overline{u_{m i n}}
\end{gathered}
$$

En (18), $\overline{y_{\max }}$ es un vector que contiene la extensión del valor máximo de las salidas $y_{\max }, \mathrm{N}$ veces; $\overline{y_{\min }}$ es un vector que contiene la extensión del valor mínimo de las salidas $y_{\min }, \mathrm{N}$ veces; $\overline{u_{\max }}$ es un vector que contiene la extensión del valor máximo de las entradas $u_{\max }, \mathrm{N}+1$ veces y $\overline{u_{m ı n}}$ es un vector que contiene la extensión del valor mínimo de las entradas $u_{\text {min }}, \mathrm{N}+1$ veces.

A continuación, se presentan los resultados obtenidos para el controlador GPC mediante simulación en Matlab, aplicando la acción de control $u_{k}$, obtenida con la optimización anteriormente planteada, a la planta no lineal, para la cual se asume que los estados no son medibles y se emplea un filtro de Kalman para realizar su estimación. Los valores de $y_{\max }, y_{\min }, u_{\max } \mathrm{y} u_{\min }$ son tomados de las Tabla II. Las matrices de sintonía para este controlador fueron:

$$
Q=\left[\begin{array}{cc}
0.001 & 0 \\
0 & 0.001
\end{array}\right] ; R=\left[\begin{array}{cc}
100 & 0 \\
0 & 100
\end{array}\right]
$$

\subsection{Respuesta del control GPC}

La Figura 10 presenta las respuesta de las salidas del sistema bajo la acción del controlador GPC para un valor inicial cercano al de estado estacionario; allí se observa cómo, a partir de aproximadamente 15 segundos, la salida $P_{g}$ tiende a su valor de referencia, con un pequeño error de estado estacionario, mientras que la salida $w_{r}$, que debería permanecer en su valor de estado estacionario, se mueve justo en el momento en el que se presenta el cambio en la referencia para $P_{g}$, lo cual indica que existe un acople entre ambas salidas. La Figura 11 describe las acciones de control bajo las mismas condiciones.

Además, de la función de costo del sistema en la Figura 12 se puede decir que se modifica en relación con los cambios en las acciones de control y en la referencia de la salida; esto se puede evidenciar en el aumento que se presenta en 10 segundos cuando hay un cambio de referencia; sin embargo, a medida que pasa el tiempo, como las acciones de control tienden a cero y la salida alcanza su referencia, la función de costo también se va a cero, o a un valor mínimo (valor del residuo $r$ ). Sin embargo, las salidas presentan error de estado estacionario $\mathrm{y}$, en este caso, la función de costo tiende a cero porque el peso de la matriz $\mathrm{Q}$, que pondera el error en la salida, es muy pequeño con respecto a la matriz $\mathrm{R}$. 
Control de potencia y velocidad de rotación de un aerogenerador usando controladores predictivos Control of power and rotation speed of a wind turbine using predictive controllers
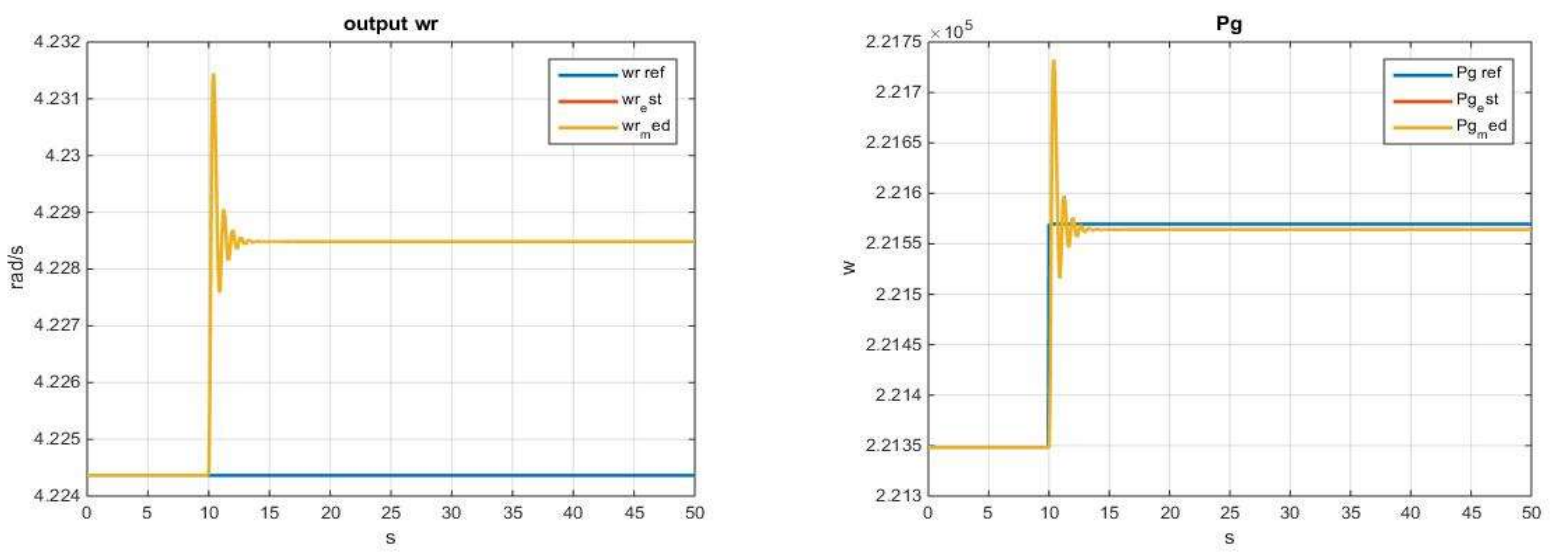

Figura 11. Respuestas de las salidas del sistema $w_{r}$ (izquierda) y $P_{g}$ (derecha) con control GPC y valor inicial cercano al valor de estado estacionario
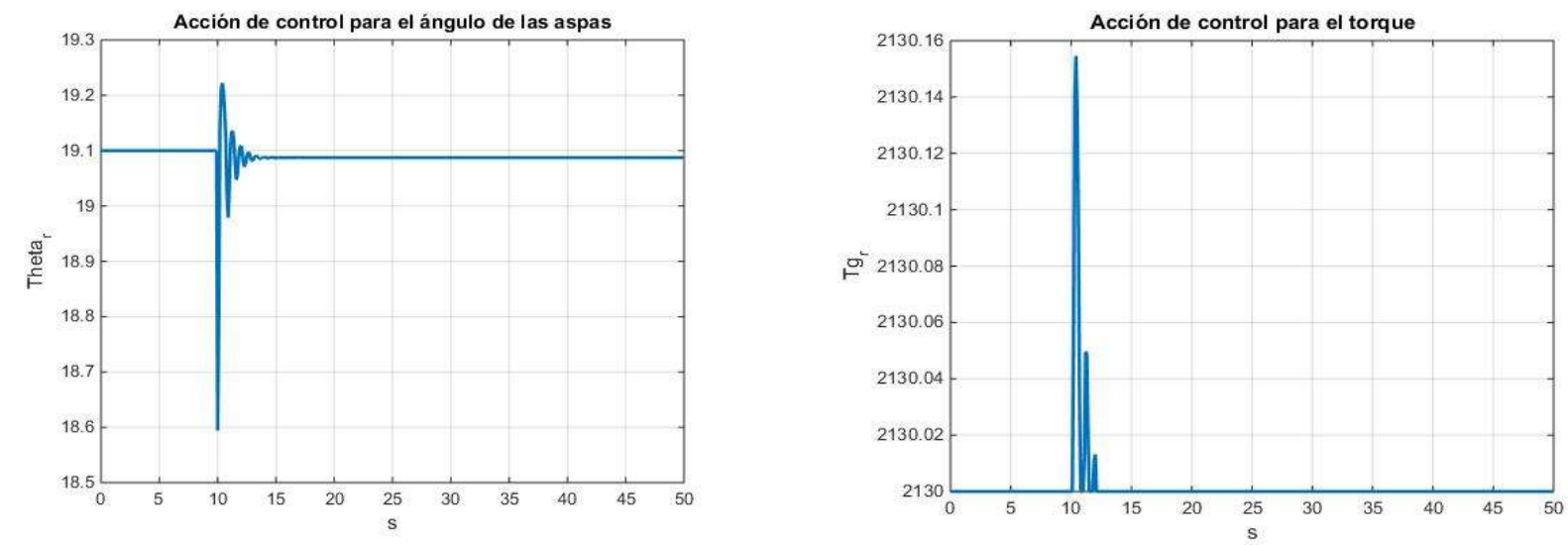

Figura 12. Respuestas de las acciones de control $\theta$ (izquierda) y $T_{g, r}$ (derecha) con control GPC y valor inicial cercano al valor de estado estacionario

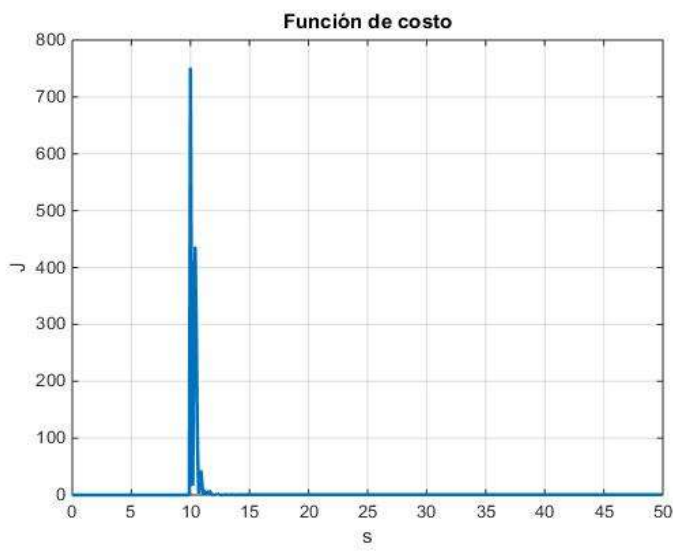

Figura 13. Funcional de costo para el control LQPC con valor inicial cercano al valor de estado estacionario

Los cambios del punto inicial anteriores son relativamente pequeños; sin embargo, si se aplican cambios porcentuales de más de un $5 \%$ de los valores de estado estacionario para $w_{r}$ y $T_{g}$ la solución para la acción de control se hace inviable, esto podría deberse a que, ante esta condición, se violan algunas restricciones en la salida del sistema. A continuación, se presenta la respuesta del GPC ante la incidencia de una perturbación, que será modelada en la velocidad del viento $v$ como ruido blanco con medio $18 \mathrm{~m} / \mathrm{s}$ y desviación estándar de 0.001 . 

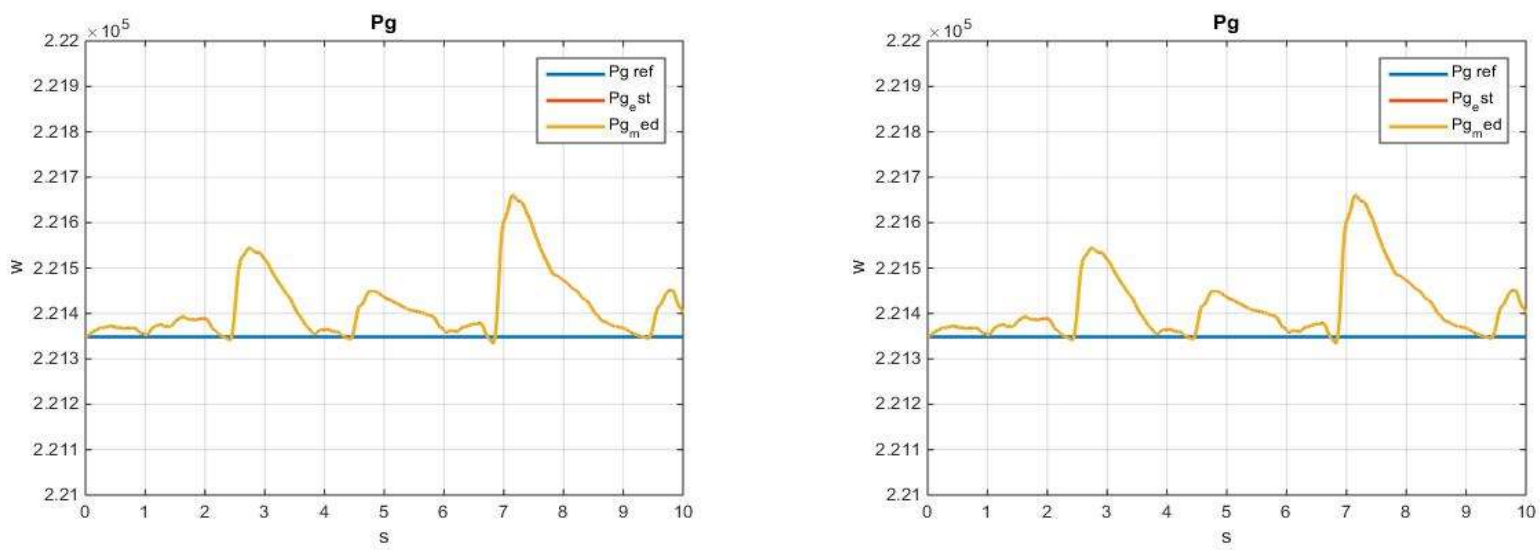

Figura 14. Respuestas de las salidas del sistema $w_{r}$ (izquierda) y $P_{g}$ (derecha) con control GPC con perturbaciones
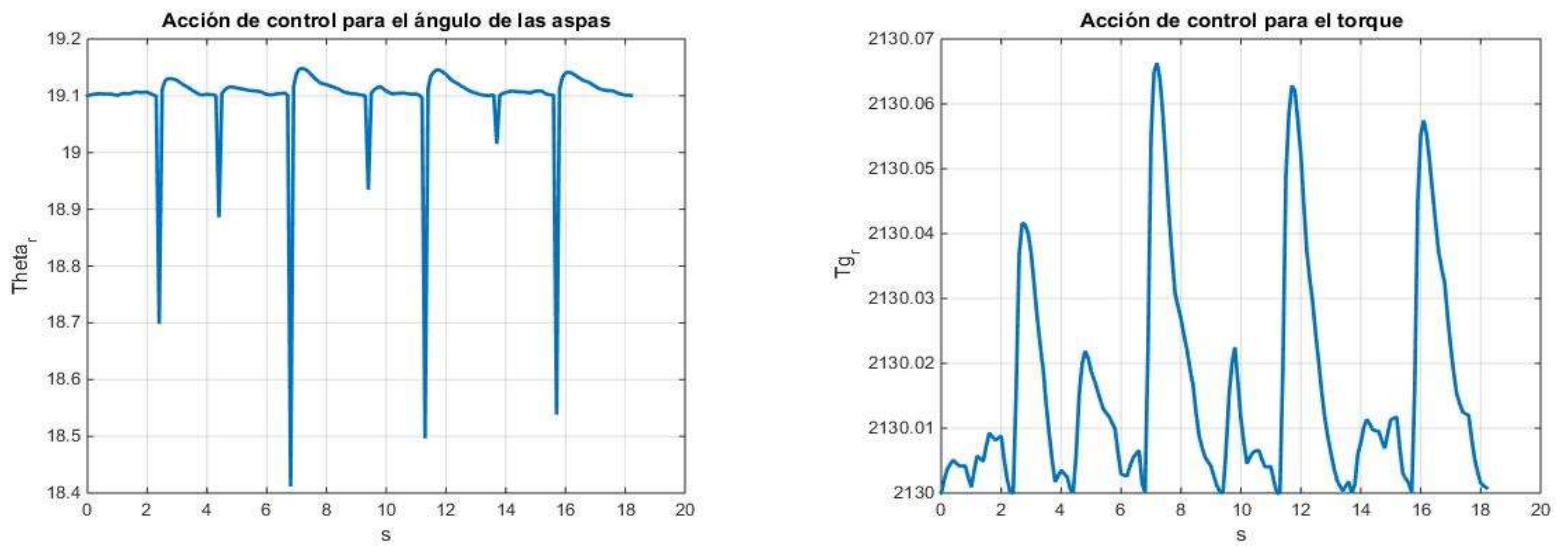

Figura 15. Respuestas de las acciones de control $\theta$ (izquierda) y $T_{g, r}$ (derecha) con control GPC con perturbaciones

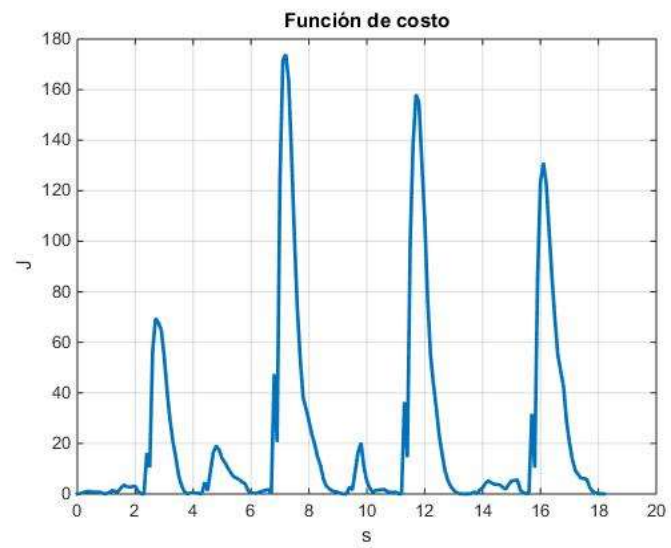

Figura 16. Funcional de costo para el control GPC con perturbaciones

Las Figuras 13, 14 y 15 presentan las salidas, las acciones de control y la funcional de costo para el sistema con controlador GPC bajo la acción de perturbaciones. De las tales respuestas se puede observar cómo el controlador GPC puede mantener la potencia de salida y la velocidad del rotor ante cambios en la velocidad del viento con un valor medio de $18 \mathrm{~m} / \mathrm{s}$ y una desviación estándar de 0.001 , y la función de costo en este caso no tiende a cero a medida que aumenta el tiempo, puesto que las acciones de control deben compensar las perturbaciones, las cuales pueden entrar en cualquier instante de tiempo; además, la salida presenta un error con respecto a la referencia, dado que la acción de control no logra compensar la perturbación en el sistema 
Control de potencia y velocidad de rotación de un aerogenerador usando controladores predictivos Control of power and rotation speed of a wind turbine using predictive controllers

inmediatamente. Además, se probaron perturbaciones con una desviación estándar superior a 0.001 ; sin embargo, la solución del problema se hizo inviable en estos casos.

\subsection{Respuesta del control GPC con restricciones adicionales}

Si se incluye una nueva restricción para la entrada del GPC que limite los cambios bruscos en ella, se tiene la misma expresión que en el controlador LQPC y presentada en las ecuaciones ( 23 ) y ( 24 ). Con la implementación de esta nueva restricción en el sistema, se obtienen las respuestas que se presentan a continuación.
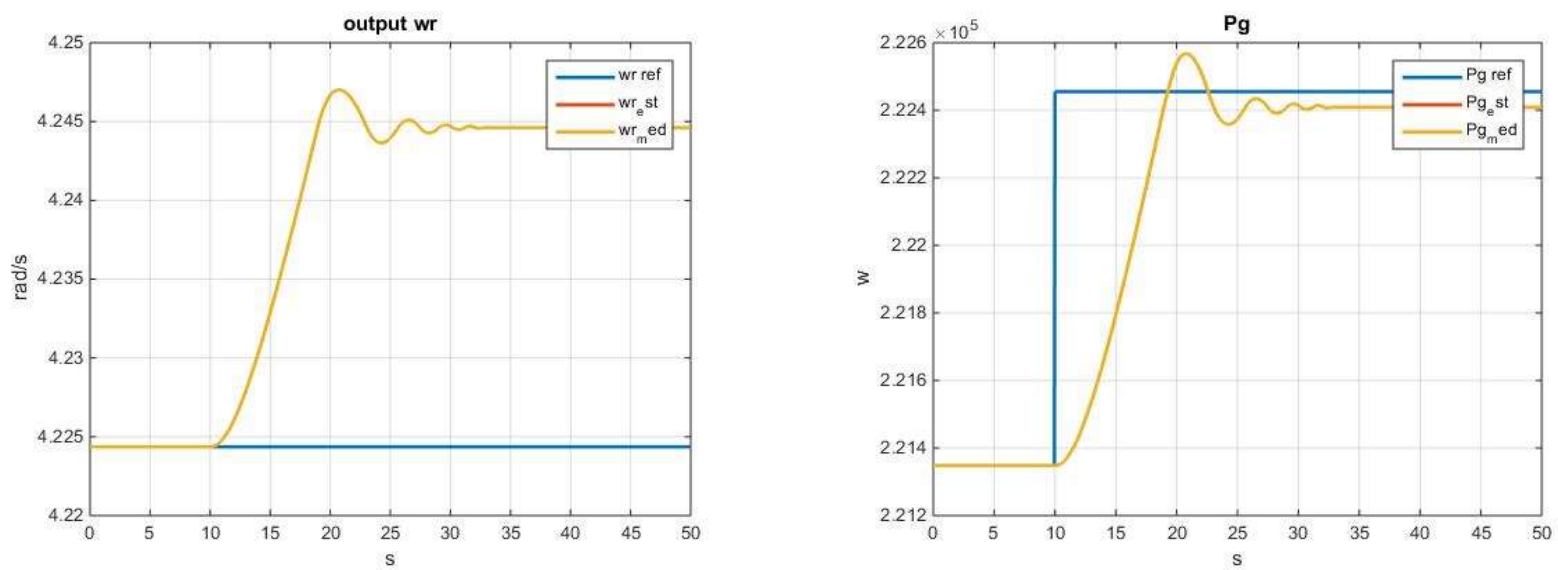

Figura 17. Respuestas de las salidas del sistema $w_{r}$ (izquierda) y $P_{g}$ (derecha) con control GPC con restricciones adicionales
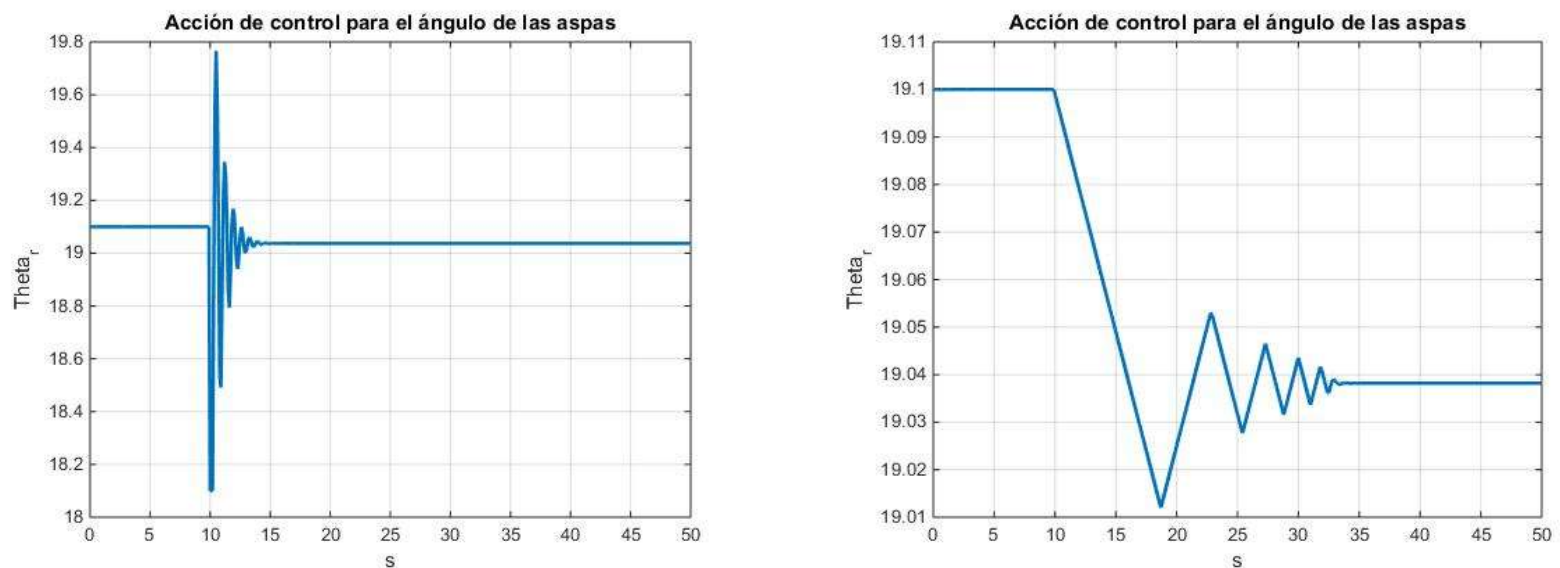

Figura 18. Respuestas de la acción de control $\theta$ para el GPC: Sin restricción en $\Delta u$ (izq.) y Con restricción en $\Delta u$. (der.) 

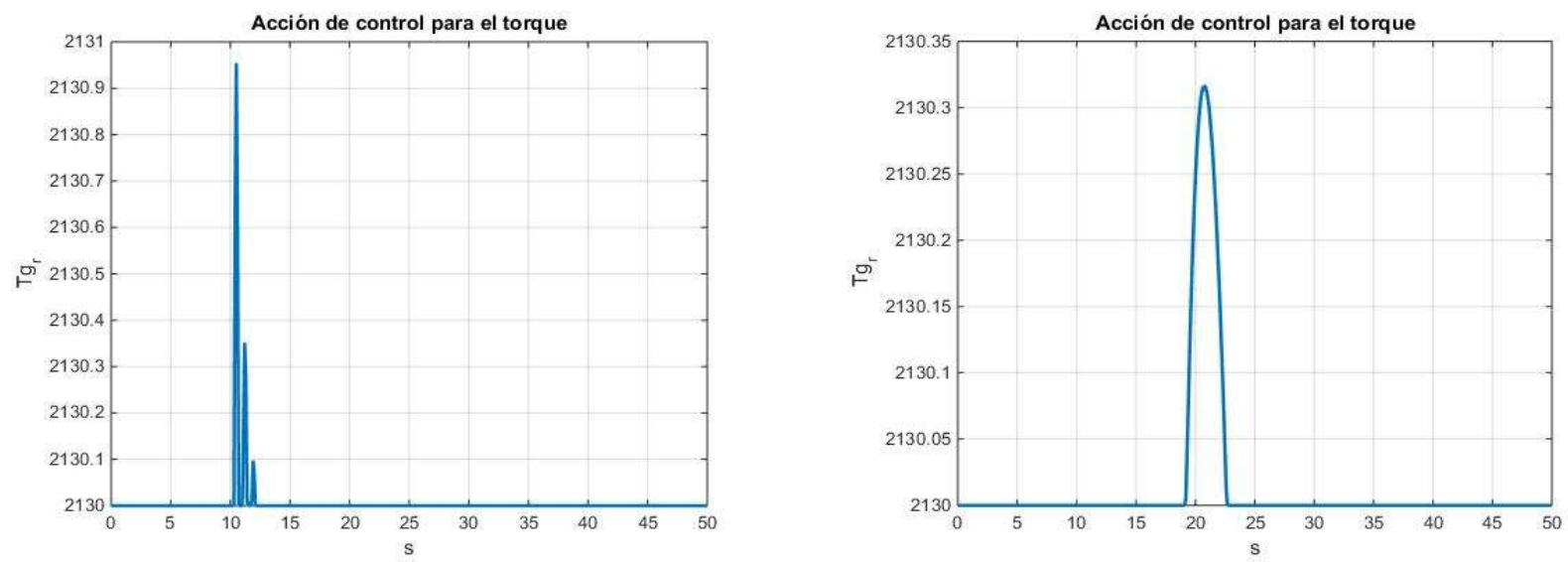

Figura 19. Respuestas de la acción de control $T_{g, r}$ para el GPC: Sin restricción en $\Delta u$ (izq.) y Con restricción en $\Delta u$. (der.)

Las Figuras 16,17 y 18 presentan las salidas, las acciones de control y la funcional de costo para el sistema con controlador GPC con restricciones adicionales. De las gráficas anteriores se puede concluir que, al añadir la restricción para el cambio en la entrada, las acciones de control se hacen más suaves; por ejemplo, en el caso de la acción de control para el ángulo de las aspas $\theta$, al imponer la restricción sobre el $\Delta u$, el cambio más brusco en la acción de control pasó de ser 1.8 a ser de 0.09 ; además, en la entrada $T_{g, r}$. no se presentan cambios bruscos como en el caso sin restricción para el cambio en la entrada, donde se presentan algunos sobrepicos. Al igual que en el LQPC, para el GPC la restricción en $\Delta u$ implica que habrá un menor esfuerzo en los componentes del sistema para alcanzar una condición deseada, sin embargo, la respuesta del sistema puede volverse más lenta al imponer esta condición, lo cual ocurre en este caso.

\subsection{Controlador GPC con eliminación de offset}

En las Figuras 10 y 16 se puede observar un error de estado estacionario en la salida con el GPC, este se puede eliminar definiendo una nueva función objetivo:

$$
\begin{aligned}
& J=\sum_{j=1}^{N}(y(k)-y r e f)^{T} Q(y(k)-y r e f)+\sum_{j=0}^{N} u(k+j)^{T} R u(k+j)+\sum_{j=0}^{N} \Delta u(k+j)^{T} S \Delta u(k+j) \\
& \text { Sujeto a: } \begin{array}{c}
\Delta u_{k} \leq \overline{y_{\max }}-\Gamma x(k+j) \\
-\Delta u_{k} \leq \Gamma x(k+j)-\overline{y_{\min }} \\
I u_{k} \leq \overline{u_{\max }} \\
-I u_{k} \leq \overline{u_{\min }} \\
|\Delta u(k+j)| \leq \Delta u_{\max }
\end{array}
\end{aligned}
$$

Además, sea:

$$
\begin{gathered}
\Delta u_{k}=\bar{I}_{b}^{-1} u_{k}-\bar{I}_{b}^{-1} \bar{I} u(k+j-1) \\
\overline{I_{b}}=\left[\begin{array}{ccc}
I & \cdots & 0 \\
\vdots & \ddots & \vdots \\
I & \cdots & I
\end{array}\right], \quad \bar{I}=\left[\begin{array}{c}
I \\
\vdots \\
I
\end{array}\right]
\end{gathered}
$$

Si se reemplaza la expresión anterior en la funcional de costo de la ecuación ( 30 ), se obtiene:

$$
\min \left(u_{k}^{T} H_{2} u_{k}+2 F_{2} u_{k}+r_{2}\right)
$$

Donde:

$$
H_{2}=H_{G P C}+{\overline{I_{b}}}^{-1^{T}}\left[\begin{array}{ccc}
S & \cdots & 0 \\
\vdots & \ddots & \vdots \\
0 & \cdots & S
\end{array}\right] \bar{I}_{b}^{-1}
$$


Control de potencia y velocidad de rotación de un aerogenerador usando controladores predictivos Control of power and rotation speed of a wind turbine using predictive controllers

$$
\begin{gathered}
F_{2}=F_{G P C}-2\left(\bar{I}_{b}^{-1} \bar{I} u(k+j-1)\right)^{T}\left[\begin{array}{ccc}
S & \cdots & 0 \\
\vdots & \ddots & \vdots \\
0 & \cdots & S
\end{array}\right] \bar{I}_{b}^{-1} \\
r_{2}=R_{G P C}+\left(\bar{I}_{b}^{-1} \bar{I} u(k+j-1)\right)^{T}\left[\begin{array}{ccc}
S & \cdots & 0 \\
\vdots & \ddots & \vdots \\
0 & \cdots & S
\end{array}\right]\left(\bar{I}_{b}^{-1} \bar{I} u(k+j-1)\right)
\end{gathered}
$$

A continuación, se presentan los resultados obtenidos al implementar este controlador, teniendo en cuenta que las matrices de sintonía $Q, R$ y $S$ fueron:

$$
Q=\left[\begin{array}{cc}
0.00001 & 0 \\
0 & 0.00001
\end{array}\right] ; R=\left[\begin{array}{cc}
0.001 & 0 \\
0 & 0.001
\end{array}\right] ; S=\left[\begin{array}{cc}
100 & 0 \\
0 & 100
\end{array}\right]
$$

Las Figuras 19, 20 y 21 presentan las salidas, las acciones de control y la funcional de costo para el sistema con controlador GPC con restricciones adicionales. En la Figura 19 se evidencia el seguimiento de la referencia por parte de las salidas; sin embargo, la salida $w_{r}$ 'presenta un pequeño error de estado estacionario que el controlador no es capaz de compensar en su totalidad. Esto se puede deber al fuerte acoplamiento que existe entre ambas salidas. No obstante, este controlador, a diferencia del GPC sin alteraciones, permite un buen seguimiento de la referencia para la salida $w_{r}$.
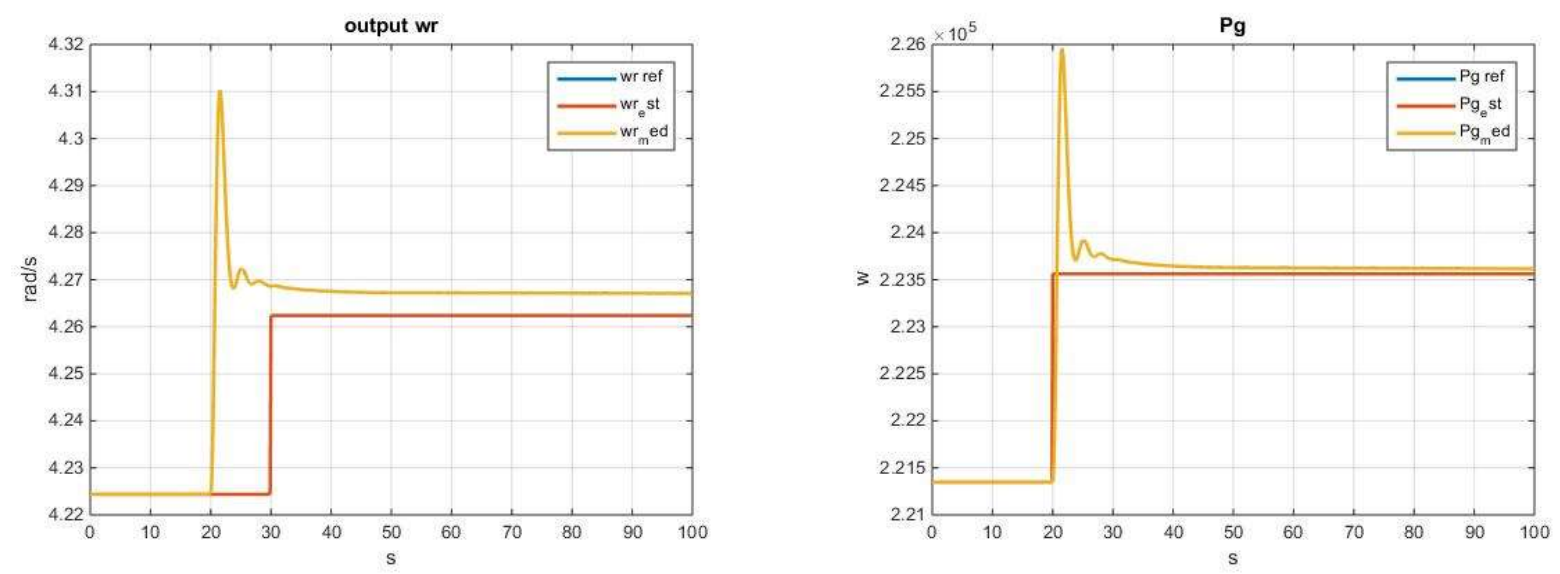

Figura 20. Respuestas de las salidas del sistema $w_{r}$ (izquierda) y $P_{g}$ (derecha) con control GPC con restricciones adicionales
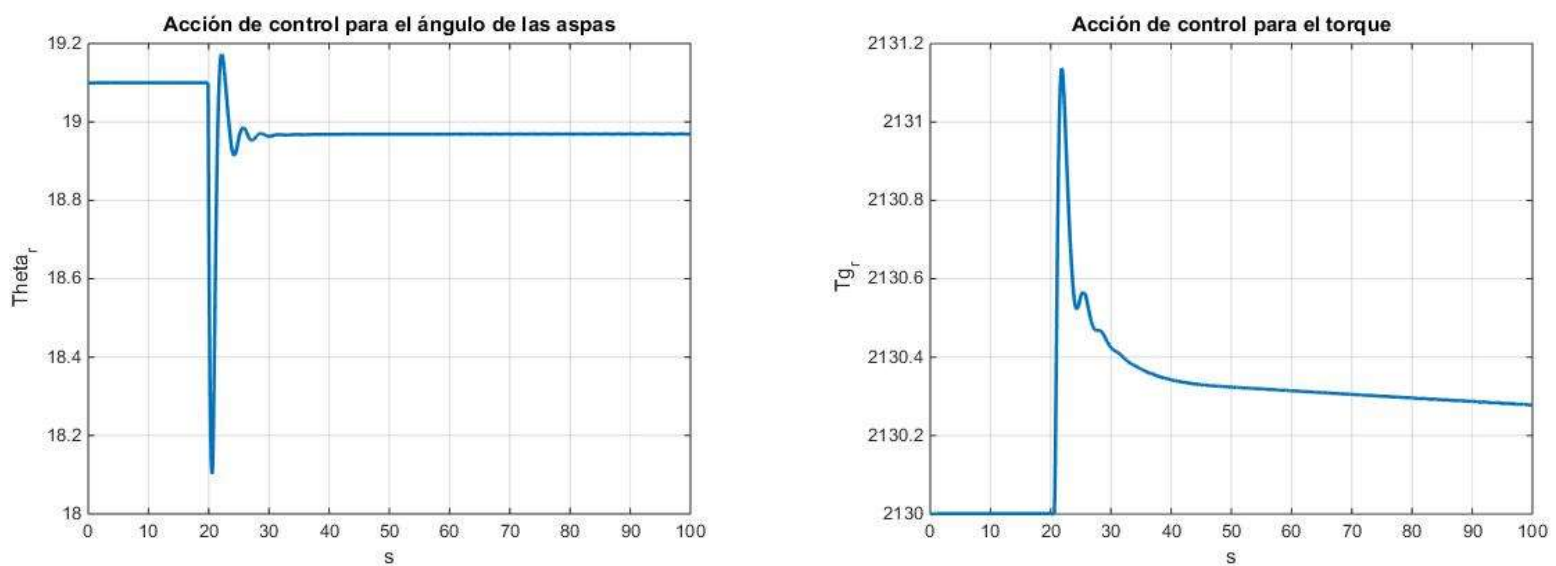

Figura 21. Respuestas de las acciones de control $\theta$ (izquierda) y $T_{g, r}$ (derecha) con control GPC con restricciones adicionales 


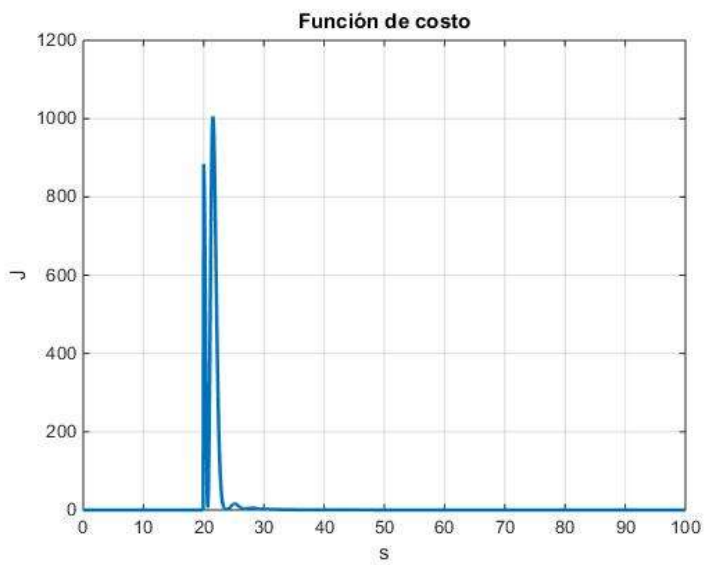

Figura 22. Funcional de costo para el control GPC con restricciones adicionales

\section{CONCLUSIONES}

En este trabajo se plantean dos estrategias de control, LQPC y GPC, para controlar la velocidad angular y el torque de un aerogenerador con el objetivo de garantizar un determinado valor para la potencia de salida de este y evitar variaciones bruscas en la velocidad angular que puedan generar desgaste o estrés mecánico. A partir de los resultados observados para las acciones de control, en los controladores LQPC y GPC, se puede decir que estas son más "suaves" para el GPC, y además se ven limitadas cuando se agrega la restricción para $\Delta u$, con lo cual se alargan los tiempos de respuesta del sistema, pero se evitan los cambios bruscos que pueden ocasionar daños en los componentes.

Para el controlador GPC se resalta el hecho de que se le debió otorgar muy poco peso, en la función de costo, al error en la salida, si no se hacía de esta manera la función de costo no decrecía o se atenuaba y, en algún punto, la solución de la optimización se hacía inviable.

\section{REFERENCIAS}

[1] E. Duque, J. Patiño, and L. Vélez, "Implementation of the ACM0002 methodology in small hydropower plants in Colombia under the Clean Development Mechanism," Int. J. Renew. Energy Res., vol. 6, no. 1, pp. $21-$ 33, 2016.

[2] I. Arto, I. Capellán-Pérez, R. Lago, G. Bueno, and R. Bermejo, "The energy requirements of a developed world," Energy Sustain. Dev., vol. 33, pp. 1-13, Aug. 2016.

[3] G. W. Arnold, "Challenges and Opportunities in Smart Grid: A Position Article," Proc. IEEE, vol. 99, no. 6, pp. 922-927, Jun. 2011.

[4] J. Cardona Gil, J. H. Gallego Orrego, C. Isaza Roldán, R. Torres Salazar, and D. López Chejne, "Integración de Tecnologías Energéticamente Eficientes en Sistemas de Climatización Operados con Energía Térmica," Rev. CINTEX, vol. 22, no. 1, pp. 83-96, 2017.

[5] M. Soshinskaya, W. H. J. Graus, J. M. Guerrero, and J. C. Vasquez, "Microgrids: experiences, barriers and success factors," Renew. Sustain. Energy Rev., vol. 40, pp. 659-672, 2014.

[6] E. Duque Grisales and J. A. Patiño Murillo, "El mercado de bonos de carbono y su aplicación para proyectos hidroeléctricos," Rev. CINTEX, vol. 18, pp. 131-143, 2013.

[7] E. A. Duque-Grisales, J. A. Patiño-Murillo, and L. D. Vélez-Gómez, "Aplicación del mercado de carbono en pequeñas centrales hidroeléctricas," Energética, vol. 44, pp. 19-32, 2014.

[8] G. Pepermans, J. Driesen, D. Haeseldonckx, R. Belmans, and W. D'haeseleer, "Distributed generation: Definition, benefits and issues," Energy Policy, vol. 33, no. 6, pp. 787-798, 2005.

[9] J. Patino, A. Marquez, and J. Espinosa, "An economic MPC approach for a microgrid energy management system," in Conference and Exposition (T\&D-LA 2014), 2014 IEEE PES Transmission \& Distribution, Medellín, Colombia, 2014, pp. 1-5.

[10] O. D. Montoya Giraldo, A. Grajales, L. F. Grisales, and C. A. Castro, "Ubicación y Operación Eficiente de Almacenadores de Energía en Micro-redes en Presencia de Generación Distribuida," Rev. CINTEX, vol. 22, no. 1, pp. 97-117, 2017. 
Control de potencia y velocidad de rotación de un aerogenerador usando controladores predictivos Control of power and rotation speed of a wind turbine using predictive controllers

[11] D. E. Olivares et al., "Trends in Microgrid Control," Smart Grid IEEE Trans. On, vol. PP, no. 99, pp. 1$15,2014$.

[12] S. Ruiz, J. Patino, A. Marquez, and J. Espinosa, "Optimal design for an electrical hybrid microgrid in Colombia under fuel price variation," Int. J. Renew. Energy Res., vol. 7, no. 24, pp. 1535-1545, 2017.

[13] J. Patino and J. Espinosa, "Control sensitivity functions of frequency regulation for a one-area power system," in 2017 IEEE 3rd Colombian Conference on Automatic Control (CCAC), 2017, pp. 1-6.

[14] S. K. Pandey, S. R. Mohanty, and N. Kishor, "A literature survey on load-frequency control for conventional and distribution generation power systems," Renew. Sustain. Energy Rev., vol. 25, no. 0, pp. 31833[4,540 NB. Dreidy, H. Mokhlis, and S. Mekhilef, "Inertia response and frequency control techniques for renewable energy sources: A review," Renew. Sustain. Energy Rev., vol. 69, pp. 144-155, 2017.

[16] N. Khezami, N. Benhadj Braiek, and X. Guillaud, "Wind turbine power tracking using an improved multimodel quadratic approach,” ISA Trans., vol. 49, no. 3, pp. 326-334, Jul. 2010.

[17] T. H. Mohamed, J. Morel, H. Bevrani, and T. Hiyama, "Model predictive based load frequency control_design concerning wind turbines," Int. J. Electr. Power Energy Syst., vol. 43, no. 1, pp. 859-867, 2012.

[18] J. Patiño, F. Valencia, and J. Espinosa, "Sensitivity Analysis for Frequency Regulation in a Two-area Power System,” Int. J. Renew. Energy Res., vol. 7, no. 2, pp. 700-706, 2017.

[19] J. Patiño, J. D. López, and J. Espinosa, "Analysis of Control Sensitivity Functions for Power System Frequency Regulation," in Applied Computer Sciences in Engineering, vol. 915, J. C. Figueroa-García, E. R. López-Santana, and J. I. Rodriguez-Molano, Eds. Cham: Springer International Publishing, 2018, pp. 606-617.

[20] F. Díaz-González, M. Hau, A. Sumper, and O. Gomis-Bellmunt, "Participation of wind power plants in system frequency control: Review of grid code requirements and control methods," Renew. Sustain. Energy Rev., vol. 34, no. 0, pp. 551-564, 2014.

[21] G. Ramtharan, N. Jenkins, and O. Anaya-Lara, "Modelling and control of synchronous generators for wide-range variable-speed wind turbines," Wind Energy, vol. 10, no. 3, pp. 231-246, 2007.

[22] H. Camblong, I. Vechiu, X. Guillaud, A. Etxeberria, and S. Kreckelbergh, "Wind turbine controller comparison on an island grid in terms of frequency control and mechanical stress," Renew. Energy, vol. 63, pp. 37-45, Mar. 2014.

[23] S. Ruiz, J. Patiño, and J. Espinosa, "Load frequency control of a multi-area power system incorporating variable-speed wind turbines," in Conference Proceedings of XVII LATIN AMERICAN CONFERENCE IN AUTOMATIC CONTROL, Medellín, Colombia, 2016, pp. 447-452.

[24] S. Ruiz, J. Patiño, and J. Espinosa, "PI and LQR controllers for Frequency Regulation including Wind Generation,” Int. J. Electr. Comput. Eng. IJECE, vol. 8, no. 5, pp. 3711-3721, 2018.

[25] E. Ikonen and K. Najim, Advanced process identification and control. New York: M. Dekker, 2002.

[26] D. W. Clarke, C. Mohtadi, and P. S. Tuffs, "Generalized predictive control—Part I. The basic algorithm," Automatica, vol. 23, no. 2, pp. 137-148, Mar. 1987.

[27] S. C. Thomsen, "Nonlinear control of a wind turbine, ME Thesis," Technical University of Denmark, 20[28] P. Li, W. Hu, R. Hu, Q. Huang, J. Yao, and Z. Chen, "Strategy for wind power plant contribution to frequency control under variable wind speed," Renew. Energy, Dec. 2017.

[29] Vestas, "General Specifications V82-1.65 MW MK II." 1996. 